\title{
Distinct evolution of infection-enhancing and neutralizing epitopes in the spike protein of SARS-CoV-2 variants (alpha, beta, gamma, delta, lambda and $\mu$ ) : a structural and molecular epidemiology study
}

\author{
Patrick Guérin ${ }^{1}$, Nouara Yahi², Fodil Azzaz², Henri Chahinian², Jean-Marc Sabatier ${ }^{3,4}$, \\ and Jacques Fantini ${ }^{2}$ \\ ${ }^{1}$ OpenHealth -CP \\ ${ }^{2}$ Aix-Marseille Univ, INSERM UMR 1072 \\ ${ }^{3}$ Aix-Marseille Univ, CNRS, INP, Inst Neurophysiopathol \\ ${ }^{4}$ Aix-Marseille University
}

November 8, 2021

\begin{abstract}
Objectives. The efficiency of Covid-19 vaccination is determined by cellular and humoral immune responses, and for the latter, by the balance between neutralizing and infection-enhancing antibodies. Here we analyzed the evolution of neutralizing and facilitating epitopes in the spike protein among SARS-CoV-2 variants. Methods. Amino acid alignments were performed on 929,203 spike sequences over the 4 last months. Molecular modeling studies of the N-terminal domain (NTD) and rod-like regions of the spike protein were performed on a representative panel of SARS-CoV-2 variants that were structurally compared with the original Wuhan strain. Results. D614, which belongs to an antibody-dependent-enhancement (ADE) epitope common to SARS-CoV-1 and SARS-CoV-2, has rapidly mutated to D614G in the first months of 2020, explaining why ADE has not been detected following mass vaccination. We show that this epitope is conformationally linked to the main ADE epitope of the SARS-CoV-2 NTD which is highly conserved among most variants. In contrast, the neutralizing epitope of the NTD showed extensive variations in SARS-CoV-2 variants. Conclusions. This molecular epidemiology study coupled with structural analysis of the spike protein indicates that the balance between facilitating and neutralizing antibodies in vaccinated people is in favor of neutralization for the Wuhan strain, alpha and beta variants, but not for gamma, delta, lambda and mu. The evolution of SARS-CoV-2 has dramatically affected the ADE/neutralization balance which is nowadays in favor of ADE. Future vaccines should consider these data to design new formulations adapted to SARS-CoV-2 variants and lacking ADE epitopes in the spike protein.
\end{abstract}

\section{Hosted file}

Guerin et al complete.pdf available at https://authorea.com/users/445096/articles/544703distinct-evolution-of-infection-enhancing-and-neutralizing-epitopes-in-the-spikeprotein-of-sars-cov-2-variants-alpha-beta-gamma-delta-lambda-and-\%C2\%B5-a-structuraland-molecular-epidemiology-study 